\title{
THE EXPECTED VALUE OF PERFECT INFORMATION IN THE OPTIMAL EVOLUTION OF STOCHASTIC SYSTEMS
}

M.A.H. Dempster

International Institute for Applied Systems Analysis, Laxenburg, Austria

RR-83-13

May 1983

Reprinted from Stochastic Differential Systems, volume 36 (1981)

Proceedings of the 3rd IFIP-WG 7/1 Working Conference, Visegrád, Hungary,

15-20 September 1980, edited by M. Arató, D. Vermes, and A.V. Balakrishnan.

INTERNATIONAL INSTITUTE FOR APPLIED SYSTEMS ANALYSIS

Laxenburg, Austria 
Research Reports, which record research conducted at IIASA, are independently reviewed before publication. However, the views and opinions they express are not necessarily those of the Institute or the National Member Organizations that support it.

Reprinted from Stochastic Differential Systems, volume 36 (1981). Pages 25-40 in Lecture Notes in Control and Information Sciences, edited by A.V. Balakrishnan and M. Thoma, IFIP. Copyright $\odot$ by Springer-Verlag Berlin Heidelberg.

All rights reserved. No part of this publication may be reproduced or transmitted in any form or by any means, electronic or mechanical, including photocopy, recording, or any information storage or retrieval system, without permission in writing from the copyright holder. 


\section{PREFACE}

Methodological research into optimization problems and techniques has a long history in the System and Decision Sciences Program at IIASA. Most recently, effort - of which this paper forms a part - has concentrated on the analysis of stochastic systems.

For a very general model of a stochastic optimization problem with an infinite planning horizon in discrete time, the author analyzes the stochastic process describing the marginal expected value of perfect information (EVPI) about the future of the system. $\mathrm{He}$ demonstrates two intuitively obvious properties of this marginal EVPI process: that its values are completely predictable at each actual decision point and that its expected values tend to decline over the future since information is potentially worth more the sooner it is available. The author is currently working on continuous time analogs of these results, which are unfortunately fraught with technical difficulties.

This work should be viewed as a theoretical prolegomenon to computational studies aimed at estimating the value of perfect or partial information in the control of stochastic systems. The central observation here is that the extra complexity and computational burden of introducing random parameters into planning or control models may sometimes be unnecessary. The (marginal) EVPI at decision points is the natural measure by which their modeling efficacy can be evaluated. 



\author{
M.A.H. Dempster \\ System and Decision Sciences, IIASA, \\ A-2361 Laxenburg, Austria \\ (On leave from Balliol College, University \\ of Oxford, Oxford OXI 3BJ, England)
}

\title{
CONTENTS
}

1. Introduction

2. The dynamic recourse problem as an abstract optimization problem

3. Characterization of optimal solutions

4. The marginal expected value of perfect information supermartingale

5. Possible extensions

6. Acknowledgements

References

\section{INTRODUCTION}

This paper uses abstract optimization theory to characterize and analyze the stochastic process describing the current marginal expected value of perfect information in a class of discrete time dynamic stochastic optimization problems which includes the familiar optimal control problem with an infinite planning horizon. Using abstract Lagrange multiplier techniques on the usual nonanticipativity constraints treated explicitly in terms of adaptation of the decision sequence, it is shown that the marginal expected value of perfect information is a supermartingale. For a given problem, the statistics of this process are of fundamental practical importance in deciding the necessity for continuing to take account of the stochastic variation in the evolution of the sequence of optimal decisions.

Let $\underset{\sim}{x}=\{\underset{\sim}{x}\}_{t=1}^{\tau}$ be a sequence of decisions in $\mathbb{R}_{n}$ and let $\underset{\sim}{\xi}=\left\{\xi_{t}\right\}_{t=1}^{\tau}$ be a discrete time stochastic process in $(\Xi, \Sigma, \mu)$ of subsequent observations. A policy (decision rule or recourse function) is a measurable map $\underset{\sim}{x}: \xi \rightarrow x(\xi)$. Consider the problem 
$(\mathrm{RP})$

$$
\inf \underset{\sim}{x} E\left[\Sigma_{t=1} \underset{\sim}{f} t \underset{\sim}{(x)}\right]
$$

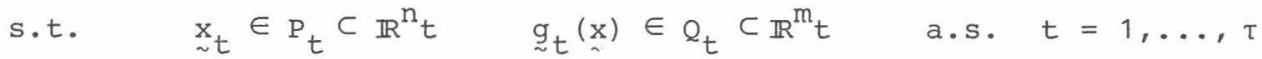

(where $n_{t} \leq n_{\tau}=n, m_{t} \leq m_{\tau}=m$ ) and the nonanticipative condition that the current decision $\underset{\sim}{\mathrm{x}}$ depends only on the sequence of observations $\xi_{1}, \xi_{2}, \ldots, \xi_{t-1}$, and realised decisions $x_{1}, x_{2}, \ldots, x_{t-1}$ (and thus $\underset{\sim}{\xi}$ ) to date. Here $f_{t}: \Xi \times x_{t=1}^{\tau} \mathbb{R}_{t}^{n_{t}} \rightarrow \mathbb{R}$ is assumed measurable in its first argument and Borel measurable in its second and $\underset{\sim}{f}(\underset{\sim}{x}):=f_{t}(\cdot, x(\cdot))$, and similarly for ${\underset{\sim}{g}}_{t} \underset{\sim}{x})$. (A full set of technical assumptions will be introduced in $\$ \$ 2$ and 3.$)$

The problem (RP)--termed the dynamic recourse problem--has a number of important applications in the mathematical sciences (cf. Dempster, 1980). Special cases include stochastic dynamic linear or quadratic programming formulations of energy-economic planning models, Birge (1980), Louveaux and Smeers $(1980,1981)$; inventory control models, see e.g. Veinott (1966); Markov decision processes with random transition matrices for manpower planning, Grinold $(1976,1980)$ and the classical discrete time optimal control model. To see the last assertion in more detail, make the following substitutions in (RP):

$$
\begin{aligned}
& \underset{\sim}{x}:=(\underset{\sim}{z}, \underset{\sim}{u}) \quad m_{t}: \equiv m \quad n_{t}: \equiv m+n \\
& Q_{t}: \equiv\{0\} \quad P_{1}:=\left\{z_{1}\right\} \times \mathbb{R}^{n} \quad P_{t}:=\mathbb{R}^{m+n} \quad t=2, \ldots, \tau
\end{aligned}
$$

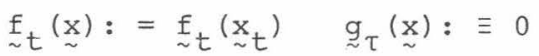

$$
\begin{aligned}
& \underset{\sim}{g}(\underset{\sim}{x}):={\underset{\sim}{z} t+1}-h\left(\underset{\sim}{z}{ }_{t}{\underset{\sim}{u} t}_{t}\right)-{\underset{\sim}{\xi} t}_{t} \quad t=1, \ldots, \tau-1 .
\end{aligned}
$$

Then (RP) reduces to the familiar control problem

$$
\sup _{\underset{\sim}{u}} E\left[\Sigma_{t=1}^{\tau} \underset{\sim t}{f}\left(\underset{\sim}{z},{\underset{\sim}{u} t}^{u}\right)\right]
$$

$$
\begin{aligned}
& \text { s.t. } \quad{\underset{\sim}{z} t+1}_{t}=h\left(\underset{\sim}{z}{ }^{\prime} \underset{\sim}{u}\right)+\underset{\sim}{\xi_{t}} \quad \text { a.s. } \quad t=1, \ldots, \tau-1 \\
& {\underset{\sim}{1}}_{1}=z_{1} \quad \text { a.s. }
\end{aligned}
$$


Control and state space constraints are easily added to (c) by suitable definition of $\mathrm{P}_{t}, t=1, \ldots, \tau$.

Characterization of the (optimal) solutions to the general problem (RP) for finite $\tau$ has been treated by Rockafellar and Wets (1976a,b, 1978) for the convex case under a slater regularity condition (constraint qualification) using the duality theory of convex conjugate functions. More recently (1981), they have given a similar treatment of the convex Bolza problem--a special case of (C)--for finite $\tau$. Hiriart-Urruty $(1978,1981)$ has considered a more general class of nonlinear special cases of (RP) for finite $\tau$. He applied a theory characterizing in terms of generalized gradients the minimum of an integral functional involving a measurable locally Lipschitz integrand subject to a measurable closed valued multifunction constraint. The version of (RP) he treated has some nondifferentiable and some differentiable constraints and a correspondingly mixed Slater/Mangasarian-Fromowitz constraint qualification is enforced.

This paper discusses the use of recently developed abstract mathematical programming theory (Dempster,1976; Zowe and Kurcyusz,1979; Brokate, 1980) to extend these results to the infinite horizon ( $\tau$ infinite) problem for the general nonlinear case under appropriate regularity conditions involving problem functions in $\mathrm{I}_{\infty}$. The support representation problem (Dempster,1976) for (RP) is addressed to obtain the appropriate stochastic maximum principle and nonanticipative supermartingale representation of the $L_{1}$ multiplier process corresponding to the nonanticipative constraints. The general results were announced in Dempster (1980); details and proofs will appear elsewhere. In this paper, emphasis is placed on precise problem formulation, results and interpretation $(\$ \$ 2$ and 3$)$ and attention is focused on the supermartingale multiplier process corresponding to the nonanticipative constraints (\$4). Technical limitations to extending the analysis of this marginal expected value of perfect information process to continuous time systems --involving say diffusion or jump dynamics--are discussed briefly in $\$ 5$ with a view to their possible relaxation in future work using recent theories of pathwise integration of stochastic differential equations (Sussman,1978; Marcus,1981).

\section{THE DYNAMIC RECOURSE PROBLEM AS AN ABSTRACT OPTIMIZATION PROBLEM}

The purpose of this section is to set up the problem (RP) as an abstract mathematical programme of the form

$$
\sup _{X \varepsilon P} f(x) \quad \text { s.t. } g(x) \in Q \quad,
$$


where $P$ and $Q$ are sets in appropriate linear topological spaces $U$ and $\mathrm{V}, \mathrm{f}: \mathrm{U} \rightarrow \mathbb{R}$ and $\mathrm{g}: \mathrm{U} \rightarrow \mathrm{V}$.

A natural assumption to make in the applications cited in $\$ 1$ is that feasible policies should be essentially bounded--i.e. in $L_{\infty}--$ on $(\Xi, \Sigma, \mu), c f$. Rockafellar and Wets $(1976,1978,1981)$, although a similar treatment of policies in $L_{p}(1 \leqq p<\infty)$ is also possible, ef. Eisner and Olsen (1975,1980), Hiriart-Urruty $(1978,1981)$. Hence assume $\Sigma$ completed with respect to $\mu$ and define

$$
L_{\infty}^{n}:=L_{\infty}\left[(\Xi, \Sigma, \mu) ; \mathbb{R}^{n}\right]
$$

and

$$
\begin{aligned}
& \mathrm{x}:=\underset{\sim}{\mathrm{x}}=\left(\underset{\sim}{\mathrm{x}} 1, \ldots, \underset{\sim \tau}{\mathrm{x}_{\tau}}\right) \\
& U:=x_{t=1} L_{\infty}^{n} \\
& P_{t}^{\bar{E}}:=\left\{\phi \varepsilon I_{\infty}^{n}{ }^{n} \mid \phi: E \rightarrow P_{t}\right\} \\
& P:=X_{t=1}^{\tau} P_{t} \Xi \\
& Q_{t}^{\Xi}:=\left\{\Psi \varepsilon I_{\infty}^{m_{t}} \mid \Psi: \Xi \rightarrow Q_{t}\right\} \\
& Q:=X_{t=1}^{\tau} Q_{t} \Xi
\end{aligned}
$$

equipping $U$ with the usual equivalent of the product topology defined in terms of the norm $\|u\|=\sup _{t}\left\|u_{t}\right\|_{\infty}$. (We shall be party to the usual abuse of terminology by referring to the equivalence class elements of Banach function spaces as functions and subject to the standard analyst/ probabilist's schizophrenia by denoting the elements of. for example, $L_{\infty}$ as both $u$ and $\underset{\sim}{u}$ depending on whether the analytic or probabilistic interpretation is to the fore.) The abstract objective function of (P) is obviously

$$
f(x):=E\left[\Sigma_{t=1} \underset{\sim t}{f} \underset{\sim}{f}(x)\right],
$$

but a little more analysis will be necessary in order to define the abstract constraint function $g$ and its range (image) space $V$.

The problem lies with the explicit characterization of nonanticipation. Its solution was first proposed in the context of stochastic Zinear programming by Eisner and Olsen $(1975,1980)$. Let $\left\{\Sigma_{t}\right\}$ be the usual increasing tower of $\sigma$-fields,

$$
\Sigma_{t} \subset \Sigma_{\tau}:=\Sigma,
$$

generated by the process $\underset{\sim}{\xi}$. Every feasible policy $\underset{\sim}{\mathrm{x}}$ is assumed to 
be $\underset{\sim}{ }$ adapted, i.e.

$$
\underset{\sim t}{x_{t}}=E{\underset{\sim}{x}}_{x_{t-1}} \mid \sum_{t} \quad \text { a.s. } \quad t=1, \ldots, \tau \text {. }
$$

After canonically embedding $L_{\infty}^{n}$ in $L_{\infty}^{n}$ in the obvious way, we may define the closed projections

$$
\Pi_{t}: L_{\infty}^{n} \rightarrow L_{\infty}^{n}, z \mapsto \Pi_{t} z:=E\left\{\underset{\sim}{z} \mid \Sigma_{t}\right\}
$$

for $t=1, \ldots, \tau$. Then $\underset{\sim}{x}$ is adapted to $\underset{\sim}{\xi}$ if, and only if,

$$
\left(2.3^{\prime}\right) \quad\left(I-\Pi_{t-1}\right) x_{t}=0 \quad t=1, \ldots, \tau \text {. }
$$

Here (when we make the usual assumption that $x_{1}$ is deterministic) $\Pi_{0}:=\Pi_{K_{1}}$, where $K_{1}$ denotes the linear span of the constants in $\mathrm{L}_{\infty}^{\mathrm{n}}{ }^{\mathrm{N}}$ embedded in $\mathrm{L}_{\infty}^{\mathrm{n}}$.

We may now define the constraint function of (P) as

$$
\mathrm{g}: \mathrm{U} \mapsto \mathrm{V}:=\mathrm{x}_{t=1}^{\tau} \mathrm{L}_{\infty}^{\mathrm{m}_{t}} \mathrm{x}\left(\mathrm{L}_{\infty}^{\mathrm{n}}\right)^{\tau}
$$

such that

$$
x \rightarrow g(x):=\left({\underset{\sim}{g}}_{1}(x),{\underset{\sim}{g}}_{2}(x), \ldots ;\left(I-\Pi_{0}\right) x_{1},\left(I-\Pi_{1}\right) x_{2}, \ldots\right),
$$

with $V$ equipped with the normed-defined equivalent of the product topology (as with $U$ ). All these considerations apply equally well to finite or infinite $\tau$. In the sequel we shall consider only the case of infinite $\tau$; necessary changes for the simpler case of finite $\tau$ are easily supplied by the reader. Further, define $\underset{\sim}{\xi^{t}}, \underset{\sim}{t}$ to be the history, i.e. ${\underset{\sim}{1}}_{1}, \xi_{2}, \ldots, \xi_{t} ; \underset{\sim}{x_{1}}, \underset{\sim}{x_{2}}, \ldots, \underset{\sim}{x}$, of the observation, respectively policy, process to time $t$. We shall specialize in what follows to the case--relevant to all the practical examples of \$1--of triangular $(R P)$, for which current constraints depend only on observation and decision histories to date, i.e.

$$
g_{t}\left(\xi_{\sim}^{t}, x_{\sim}^{t}\right) \varepsilon Q_{t}
$$

a.s. $t=1,2, \ldots$.

(Thus the measurability properties for the $g_{t}$ cited in $\$ 1$ may be restricted to $\Sigma_{t}$ rather than $\Sigma$.) 
This completes the basic set of assumptions needed to specify and analyze the dynamic recourse problem (RP) over an infinite horizon. Further(illustrative) technical assumptions will be introduced as required to complete the analysis in $\$ 3$.

\section{CHARACTERIZATION OF OPTIMAL SOLUTIONS}

The characterization of optimal solutions for the abstract mathematical programming problem (P) necessitates consideration (perhaps only implicitly) of its Langrangian function

$$
\phi\left(x, y^{\prime}\right):=f(x)+y^{\prime} g(x),
$$

for $\mathrm{x} \in \mathrm{U}$ and multiplier vectors $\mathrm{y}^{\prime} \varepsilon \mathrm{V}^{\prime}$, the dual space of $\mathrm{V}$ (consisting of all linear functionals on $V$ continuous in the given topology for $V$ ).

We shall thus here need the following characterization of $L_{\infty}^{*}$, due essentially to Yosida and Hewitt (1952) (see also Dubovitskii and Milyutin, 1965, Valadier, 1974, and Dempster, 1976). A finitely additive row n-vector valued measure $\pi^{\prime}: \Sigma \rightarrow \mathbb{R}^{n^{\prime}}$ on $(\Xi, \Sigma)$ is purely finitely additive $\left(p, f \cdot a_{.}\right)$if, and only if, for all countably additive real valued measures $\nu$ on $(\Xi, \Sigma)$ and for all $\varepsilon>0$ there exists $A_{\varepsilon} \varepsilon \Sigma$ such that $\left|\nu\left(A_{\varepsilon}\right)\right|<\varepsilon$ and $\pi^{\prime}\left(A_{\varepsilon}\right)=0^{\prime} \varepsilon \mathbb{R}^{n^{\prime}}$, i.e. a p.f.a. measure is carried by sets assigned arbitrarily small measure by any countably additive measure. (Prime is used to denote a dual element; in the finite dimensional case this is consistent with vector transposition.) Denote by $\mathrm{L}_{\infty}^{\mathrm{n}^{*}}$ the (Banach) dual space of $L_{\infty}^{n}$ (as defined in (2.1)), by $L_{1}^{n^{\prime}}$ the space of (coordinatewise) absolutely integrable row n-vector valued functions on $(\Xi, \Sigma)$ and by $\mathrm{P}^{\mathrm{n}^{\prime}}$ the space of purely finitely additive row n-vector valued measures on $(\Xi, \Sigma)$.

Proposition: 3.1. Given the measure space $(\Xi, \Sigma, \mu)$, if $\Sigma$ is complete with respect to the $\sigma$-finite measure $\mu$, then

$$
L_{\infty}^{n^{*}} \cong L_{1}^{n^{\prime} \oplus} P^{n^{\prime}}
$$

Here $\cong$ denotes isometric isomorphism and the action of $y^{\prime} \varepsilon L_{\infty}^{n^{*}}$ on an $\mathrm{n}$-vector valued function $\mathrm{x} \varepsilon \mathrm{L}_{\infty}^{\mathrm{n}}$ is given by

$$
\begin{aligned}
\mathrm{Y}^{\prime} \mathrm{x} & :=\delta_{\Xi} \mathrm{Y}^{\prime}(\xi) \mathrm{x}(\xi) \mu(\mathrm{d} \xi)+\int_{\Xi} \pi^{\prime}(\mathrm{d} \xi) \mathrm{x}(\xi) \\
& :=\mathrm{y}_{1}^{\prime} \mathrm{x}+\mathrm{y}_{2}^{\prime} \mathrm{x} .
\end{aligned}
$$


The first integral in (3.2) is simply an abstract Lebesque integral; the second requires the analogous integration theory developed for finitely additive measures by Dunford and Schwartz (1956). In fact, Valadier (1974) extended the result of Proposition 3.1. to the $\sigma$-finite case from the finite case established by Yosida and Hewitt (1952), while Dubovitskii and Milyutin (1967) independently gave a complete treatment of $L_{\infty}^{*}$ in terms of singular functionals ( $y_{2}^{\prime}$ of (3.2)) without reference to their integral representations. A finer characterization of $L_{\infty}^{*}$ in terms of natural subspace of $\mathrm{P}^{\mathrm{n}^{\prime}}$ appears in Dempster (1976).

We are now in a position to make precise sense of the Langrangian function (3.1) for (P). According to (2.4) we are interested in representation of the dual space, $x_{t=1}^{\infty} L_{\infty}^{m_{t}^{*}} \times\left(L_{\infty}^{n}\right)^{\mathbb{N}}$, of $V\left(=x_{t=1}^{\infty} L_{\infty}^{m_{t}} \times\left(L_{\infty}^{n}\right) \mathbb{N}^{\mathbb{N}}\right)$, where $\mathbb{N}$ denotes the natural numbers. A straightforward application of Proposition 3.1 yields $\phi$ as given by

$$
\begin{aligned}
& \phi\left(x, y^{\prime}\right) \\
& =\sum_{t=1}^{\infty}\left\{\operatorname{Ef}_{\sim t}(\underset{\sim}{x})+E_{\sim}^{y}{\underset{\sim}{g} t}_{\sim}^{\prime}(\underset{\sim}{x})+\operatorname{Es}_{\sim}^{\prime}\left(I-\Pi_{t-1}\right) \underset{\sim t}{x}\right. \\
& +\int_{\Xi} \pi_{t}^{\prime}(d \xi) g_{t}(\xi, x(\xi)) \\
& +\int_{\Xi} \Psi_{t}^{\prime}(d \xi)\left[x_{t}(\xi)-E\left\{x_{t} \mid \Sigma_{t-1}\right\}(\xi)\right] \\
& +\int_{\Xi \times \mathbb{N}} x_{\infty}^{\prime}(d \xi, d t)\left(g_{t}(\xi, x(\xi)), \underset{\sim t}{x_{t}}(\xi)-E\left\{\underset{\sim t}{x} \mid \Sigma_{t-1}\right\}(\xi)\right)
\end{aligned}
$$

using the fact (Yosida and Hewitt, 1952) that all p.f.a. measures on $\mathbb{N}$ (with counting measure \# taken as ground measure) are carried by neighbourhoods of $\infty$. In (3.3)

$$
\underset{\sim}{y_{t}^{\prime}} \in L_{\infty}^{n_{t}{ }^{\prime},} \underset{\sim}{\rho} \rho_{t}^{\prime} \varepsilon L_{\infty}^{m_{t}{ }^{\prime},} \pi_{t}^{\prime} \varepsilon P^{n_{t}{ }^{\prime},} \Psi_{t}^{\prime} \varepsilon P^{n^{\prime},}
$$

$\chi_{\infty}^{\prime} \varepsilon P\left[(\Xi \times \mathbb{N}, \varepsilon \times \gamma(\mathbb{N}), \mu \times \#) ; \mathbb{R}^{m+n^{\prime}}\right]$, the space of row (m+n)-vector valued measures on the product $\sigma-f i e l d$ shown, and in the corresponding integral $g_{t}$ has been canonically embedded in $\mathbb{R}^{m}$.

Next we characterize an optimum $x_{0}$ of (P) in terms of a suitable concept of derivatives of the Lagrangian $\phi$ given by (3.3). Rather than use minimal concepts and introduce highly technical conditions on (P), we shall by way of illustration use Fréchet derivatives and give regularity conditions only for (RP) sufficient to ensure the truth of the following Kuhn-Tucker Theorem for (P), ef. Zowe and Kurcyusz (1979). 
Suffice it to say here that versions of Proposition 3.2 below are available involving both generalized derivatives ( $f$. Hiriart-Urruty, 1981) and (one-sided) Gateaux directional derivatives (Dempster, 1976) under minimal regularity conditions ( $c f$. Dempster, 1976; Brokate, 1980) for (P) posed in locally convex Hausdorff topological vector spaces. We shall need the concept of the dual cone $Q^{\prime} \subset V^{\prime}$ of a set $Q \subset V$ as

$$
Q^{\prime}:=\left\{y^{\prime} \varepsilon V^{\prime}: y^{\prime} z \geqq 0, \forall z \varepsilon Q\right\} \quad,
$$

and similarly for sets in $U$.

Proposition: 3.2. Let $U$ and $V$ be Banach spaces and the problem functions $f$ and $g$ of $(P)$ be Fréchet differentiable with derivatives $\nabla f$ and $\nabla g$ respectively. Then under suitable regularity conditions on (P), $\mathrm{x}_{0}$ an optimum for (P) implies that there exists $y_{0}^{\prime} \varepsilon Q^{\prime}$ such that

$$
\omega_{0}^{\prime}:=\nabla_{\mathrm{x}} \phi\left(\mathrm{x}_{0}, \mathrm{y}_{0}^{\prime}\right)=\nabla \mathrm{f}\left(\mathrm{x}_{0}\right)+\mathrm{y}_{0}^{\prime} \nabla \mathrm{g}\left(\mathrm{x}_{0}\right) \varepsilon-\mathrm{P}^{\prime}
$$

$$
\begin{array}{ll}
\mathrm{g}\left(\mathrm{x}_{0}\right)=\nabla_{\mathrm{y}}, \phi\left(\mathrm{x}_{0}, \mathrm{y}_{0}^{\prime}\right) \varepsilon \mathrm{Q} \\
\omega_{0}^{\prime} \mathrm{u} \leqq \omega_{0}^{\prime} \mathrm{x}_{0} & \forall \mathrm{u} \varepsilon \mathrm{P} \\
\mathrm{y}_{0}^{\prime} \mathrm{g}\left(\mathrm{x}_{0}\right) \leqq \mathrm{y}_{0}^{\prime} \mathrm{v} & \forall \mathrm{v} \varepsilon \mathrm{Q}
\end{array}
$$

Conditions (3.5) are termed Kuhn-Tucker (necessary) conditions for an optimum of (P).

If $0 \varepsilon \mathrm{P} \subset \mathrm{U}$ and $0 \varepsilon \mathrm{Q} \subset \mathrm{V}$, the last two imply the complementary slackness conditions

$$
\omega_{0}^{\prime} x_{0}=0 \quad y_{0}^{\prime} g\left(x_{0}\right)=0
$$

Applying Proposition 3.2 under suitable regularity assumptions on (RP) yields a (necessary) characterization of its optimal policies in terms of the abstract Lagrangian of (P) given by (3.3). However, inspection of (3.3) raises the question of conditions under which this characterization remains valid if the awkward terms involving integrals with respect to purely finitely additive measures are dropped. This is a special case of the general support representation problem (Dempster, 1976) which has appeared in the control (Dubovitskii and Milyutin,1965), economics (e.g. Prescott and Lucas,1972) and optimization (Rockafellar and Wets, 1976a,b,1978) literature. 
To solve the support representation problem for (RP) we must give conditions on the problem sufficient to make both the stochastic p.f.a. measures $\pi_{t}^{\prime}$ and $\psi_{t}^{\prime}, t=1,2, \ldots$, and the intertemporal p.f.a. measure $x_{\infty}^{\prime}$ of (3.3) vanish. The following conditions are a distillation of the literature cited above. Some terms and definitions will be needed. A policy history $\underset{\sim}{x^{t}}$ is termed feasible if it satisfies the constraint structure of (RP) to time $t$, i.e. if we have for its components

$$
\underset{\sim S}{x} \in P_{S} \quad{\underset{\sim}{g}}_{s}\left({\underset{\sim}{x}}^{s}\right) \in Q_{s} \quad \text { a.s. } \quad s=1, \ldots, t .
$$

Define

$$
x_{t}:=\left\{x_{t} \in \mathbb{R}^{n}: x_{t}=x_{t}(\xi), \xi \in \underset{\Xi}{ }, \underset{\sim}{x^{t}} \text { feasible }\right\}
$$

and

$$
C_{t}:=\left\{x_{t} \in \mathbb{R}^{n}{ }^{n}: x_{t}=x_{t}(\xi), \xi \in \Xi, \underset{\sim}{x} \text { feasible }\right\} .
$$

clearly $C_{t}$ essentially lies in $x_{t}$, but the analytical (computational) intractability of (RP) arises from the fact that this inclusion is in general essentially strict. The controllability (or relatively complete recourse) condition

$$
\left.\mu\right|_{\Sigma_{t}}\left(x_{t} \Delta C_{t}\right)=0 \quad t=1,2, \ldots,
$$

ensures almost sure decision recourse at all times from any realization of the observation (and decision) process to date and forces the optimal stochastic p.f.a. measure $\pi_{t}^{\prime}$ of Proposition 3.2 to vanish. Rockafellar and Wets (1976a) obtain more technical sufficient conditions. They show by example that, without the explicit introduction into the problem of the constraints which bind at an optimum induced on $c_{t}$ by later stages, the support representation problem in terms of $L_{1}$ multipliers for (RP) is insoluble. Since a nonanticipative constraint (2.3) cannot lead to infeasibilities of subsequent nonanticipative constraints, we can conclude immediately that the stochastic p.f.a. measures $\psi_{t}^{\prime}, t=1,2, \ldots$ always vanish at the optimum. To ensure that the optimal intertemporal p.f.a. measure $x_{\infty}^{\prime}$ vanishes, it suffices to assume that $0 \in \mathrm{P}_{t^{\prime}} t=1,2, \ldots$ and the finite horizon approximation condition:

$$
\begin{aligned}
& \text { for some } \tau(\geqq 1), \underset{\sim}{x} \text { feasible implies }(\underset{\sim}{x}, 0,0, \ldots) \\
& \text { feasible for } a l l t \geqq \tau .
\end{aligned}
$$

Examples of nontrivial p.f.a. measures (in the absence of this condition) 
are known (see Prescott and Lucas,1972).

Next we must state suitable regularity conditions on (RP) sufficient to ensure the conclusions of Proposition 3.2. in terms of $L_{1}$ multipliers. Hence we assume (by way of illustration) that $P_{t} \subset \mathbb{R}_{t}$ and $Q_{t} \subset \mathbb{R} m_{t}$ are closed convex cones, $0 \in Q_{t}$ and the problem functions $f_{t} g_{t}$ are differentiable with respect to their policy components with gradients $\nabla f_{t^{\prime}}, \nabla g_{t^{\prime}}$ $t=1,2, \ldots$. Given a set $C$ (in a linear topological space) and a point $x \in C$ define the inner approximation cone

$$
I(x ; C):=\cap \boldsymbol{p}_{\lambda \geq 0} \lambda(\mathrm{C}-\mathrm{x}) \quad,
$$

where the intersection is taken over neighbourhoods $\mathcal{N}$ of $\mathrm{x}$. We shall assume that for an optimal policy $\underset{\sim 0}{\mathrm{x}}$

$$
\underset{\sim}{g}\left(\underset{\sim 0}{\left(x_{0}^{t}\right)}+\nabla \underset{\sim}{g} \underset{\sim 0}{\left(x_{0}^{t}\right)} I\left(\underset{\sim}{x}, x_{\tau=1}^{t} P_{\tau}\right)-Q_{t}=\mathbb{R}^{m}\right.
$$

$$
\text { a.s. } \quad t=1,2, \ldots
$$

Since the (linear) projections $I-I_{t}$ of (2.3') are closed, (3.9) is sufficient to ensure that the abstract problem function $g$ of (2.4) satisfies

$$
g\left(x_{0}\right)+\nabla g\left(x_{0}\right) I\left(x_{0} ; P\right)-Q=V
$$

in terms of its Fréchet derivative at an optimum $x_{0}$ of (P) for the closed convex cone $\mathrm{Q} \subset \mathrm{V}, \mathrm{cf}$. Dempster (1976), Zowe and Kurcyusz (1979). Finally we are in a position to apply Proposition 3.2 to obtain a local version of the Kuhn-Tucker necessary conditions (3.5) for an optimal policy $\underset{\sim 0}{x_{0}}$ of $(R P)$, in terms of a stochastic maximum principle involving the $L_{1}$ multiplier processes $\underset{\sim}{y^{\prime}}$ and $\rho_{\sim}^{\prime}$, of the form

$$
\begin{aligned}
& Y_{t}^{\prime} \varepsilon \quad Q_{t}^{\prime} \quad \text { a.s. }
\end{aligned}
$$

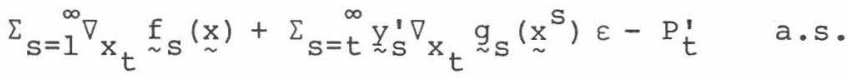

(3.11) $\quad \underset{\sim}{x} \varepsilon P_{t} \quad \underset{\sim}{g}(\underset{\sim}{x}) \varepsilon Q_{t} \quad \underset{\sim}{x}=E\left\{\underset{\sim}{x} \mid \sum_{t-1}\right\} \quad$ a.s.

$$
\begin{aligned}
& E\left[\sum_{S=1}^{\infty} \nabla_{x_{t}} \underset{\sim S}{f} \underset{\sim}{(x)}+\sum_{\tau=t}^{\infty} \underset{\sim}{y} \nabla^{\prime} x_{t} \underset{\sim S}{g} \underset{\sim}{\left(x^{S}\right)}\right] \underset{\sim t}{x_{t}}=0 \\
& E \rho_{t}^{1}\left(\underset{\sim t}{x}-E\left\{\underset{\sim t}{x} \mid \Sigma_{t-1}\right\}\right)=0 \text { for } t=1,2, \ldots \text {. }
\end{aligned}
$$


In the case that all problem functions are concave in the policy variables, conditions (3.11) are also sufficient--in general, they are not. In the next section, we turn to an analysis and interpretation of the (optimal) multiplier process $\rho_{\sim}^{\prime}$ corresponding to the nonanticipative constraints $(2.3)$ of (RP).

\section{THE MARGINAL EXPECTED VALUE OF PERFECT INFORMATION SUPERMARTINGALE}

In this section we shall assume that a fixed optimal policy $\underset{\sim}{x_{0}}$ for (RP) is specified. (Various further assumptions may be adduced to the problem to guarantee existence and even uniqueness of the optimal policy, but these will not concern us here.) We shall apply modern perturbation theory for the abstract programme (P) of $\$ 2$, see e.g. Lempio and Maurer (1980), to study the nonanticipative constraint multiplier process $\rho^{\prime}$ corresponding to the chosen optimal policy $\underset{\sim}{x_{0}}$ for (RP). Of interest are perturbations to the nonanticipative constraints (2.3) of the form

$$
\underset{\sim t}{x}=E\left\{\underset{\sim}{x} \mid \sum_{t-1}\right\}-{\underset{\sim}{z} t}
$$$$
\text { a.s. } \quad t=1,2, \ldots \text {, }
$$

where the $z_{t}$ are arbitrary $n$-vector valued functions measurable with respect to $\Sigma_{s(t)}(s(t)>t)$ and hence representing information on the future of the observation process $\underset{\sim}{\xi}$. More specifically, $f i x$ and an arbitrary $n$-vector valued function $z_{t}$ measurable with respect to $\Sigma_{s}$ for some fixed $s>t$ and consider perturbations of the $t^{\text {th }}$ nonanticipative constraint of the form

$$
\left.\underset{\sim t}{x}=\underset{\sim t}{x} \mid \Sigma_{t-1}\right\}-\alpha \underset{\sim t}{z}
$$

a.s.

for $\alpha \varepsilon[0, \delta](\delta>0)$. Denote the optimization problem resulting from the perturbation $(4.2)$ as (RP $\left.\left[\alpha z_{t}\right]\right)$ and its abstract equivalent as $\left(P\left[\alpha z_{t}\right]\right)$. Define the abstract perturbation function $\pi: V \rightarrow \mathbb{R} \cup\{\infty\}$ as

$$
\pi\left(\alpha z_{t}\right):=\sup \left\{f(x): x \in P, g(x)+\alpha\left(0, \ldots, 0, z_{t}, 0, \ldots\right) \varepsilon Q\right\}
$$

Then, under conditions on the problem data of (RP) such that the original problem has an optimal solution, the perturbed problems $P\left[\alpha z_{t}\right]$ will have feasible solutions. We shall assume that we may find a curve $x(\alpha)$ such that $x(\alpha)$ is feasible for $P\left[\alpha z_{t}\right]$ and $l i m_{\alpha \downarrow 0} x(\alpha)=x_{0} \varepsilon U$. Then, since the closed projection $\left(I-I_{t}\right)$ defines a subspace of $L_{\infty}$, the Lagrange multiplier $\rho_{t}^{\prime} \varepsilon L_{1}^{n^{\prime}}$ for the constraint (2.3) is an anihilator (supporting hyperplane) of this subspace. Under our assumptions (applying Theorem 4.3, Lempio and Maurer,1980) we may thus conclude that we 
may choose

$(4.4)$

$$
\rho_{t}^{\prime}=\nabla_{t} \pi(0)
$$

where $\nabla_{t} \pi$ denotes the Fréchet derivative of the perturbation function (4.3) of the abstract problem (P) evaluated at 0 under perturbations of the form (4.2) at time $t$. That is, the current state $\rho_{t}^{\prime}$ of the nonanticipative constraint multiplier process $\rho_{t}^{\prime}$ in $L_{1}^{n}$ represents the marginal expected value of perfect information (EVPI) at time $t$ with respect to future states of the observation process $\underset{\sim}{\xi}$.

We first establish that this marginal EVPI process $\rho{ }^{\prime}--1 i k e$ the optimal policy process $\underset{\sim 0}{x_{0}}$ itself--is adapted to the observation process $\underset{\sim}{\xi} \cdot$

Lemma: 4.1. The process $\rho^{\prime}$ in $\mathrm{L}_{1}^{\mathrm{n}^{\prime}}$ is nonanticipative, i.e.
$(4.5)$

$$
\left.\rho_{t}^{\prime}=E{\underset{\sim}{\rho}}_{t}^{\prime} \mid \Sigma_{t-1}\right\}
$$$$
\text { a.s. } \quad t=1,2, \ldots
$$

This fact follows from the observation that expression (4.4) for $\rho_{t}^{\prime}$ does not depend on any particular perturbation (4.2) representing some future knowledge of the observation process $\underset{\sim}{\xi}$.

Next we show that the process $\underset{\sim}{\rho}$ has the supermartingale property. This reflects the fact that the earlier information on the future observation process $\underset{\sim}{\xi}$ is available, the more its marginal expected worth to optimal decision making.

Theorem: 4.2. The process $\rho_{\sim}^{\prime}$ in $L_{n}^{1}$, is a supermartingale, i.e.

$$
\rho_{t}^{\prime} \geq E\left\{\rho_{s}^{\prime} \mid \Sigma_{t}\right\} \quad \text { a.s. for }(1 \leqq) \quad t<s \text {. }
$$

By virtue of (4.5) we must show for fixed $t$ and $s>t$ that

$$
E\left\{\rho_{t}^{\prime} \mid \Sigma_{t-1}\right\} \geqq E\left\{\rho_{s}^{\prime} \mid \Sigma_{t}\right\} \text { a.s. }
$$

But a further consequence of (4.5) is that for all $s \geqq t$

$$
E\left\{\rho_{t}^{\prime} \mid \Sigma_{s}\right\}=E\left\{\rho_{t}^{\prime} \mid \Sigma_{t-1}\right\} \text { a.s. , , }
$$

and hence $(4.6)$ is equivalent to showing that

$$
E\left\{\rho_{t}^{\prime} \mid \Sigma_{t}\right\} \geqq E\left\{\rho_{s}^{\prime} \mid \Sigma_{t}\right\} \quad \text { a.s. }
$$


But information on the future of $\xi$ after time s-1, as represented by an $n$-vector valued perturbation function $z$ measurable with respect to $\Sigma_{\mathrm{u}}$ for $\mathrm{u} \geqq \mathrm{s}$, cannot be worth less in expectation the earlier it is known, i.e.

$$
\pi\left(\alpha z_{t}\right) \geqq \pi\left(\alpha z_{s}\right)
$$

where $z_{s}:=z_{t}:=z$. Indeed, an optimal policy for the problem perturbed at time $t$ can take this information into account earlier than a corresponding policy for the problem perturbed at $\mathbf{s}$. Hence, subtracting $\pi(0)$ from each side of (4.7), dividing by $\alpha>0$ and passing to the limit as $\alpha \rightarrow 0$, yields

$$
\rho_{t}^{\prime}=\nabla_{t} \pi(0) \geqq \nabla_{s} \pi(0)=\rho_{s}^{\prime}
$$

Since integration is nonnegativity preserving

$$
E\left\{\sim_{t} \mid \Sigma_{t}\right\} \geqq E\left\{\sim_{s} \mid \Sigma_{t}\right\} \quad \text { a.s. }
$$

\section{POSSIBLE EXTENSIONS}

As noted in the introduction, the marginal expected value of perfect information (EVPI) process $\rho_{\sim}^{\prime}$ is of considerable potential importance for stochastic systems of the dynamic recourse type arising in practice (see $\$ 1$ ). If this nonanticipative supermartingale process in $L_{1}^{n^{\prime}}:=$ $L_{1}\left[(\Xi, \Sigma, \mu) ; \mathbb{R}^{n^{\prime}}\right]$ remains in a ball of (problem dependent) radius $\varepsilon>0$ for all $t$ after some time $s \geqq 1$, then the stochastic elements of the problem are practically inessential from time $s$ onward and a deterministic model--and simpler computational procedure--should suffice. of course, this statement raises the knotty problems of prior numerical computation of the marginal EVPI process, or--more realistically--of bounds on this process, etc. (in this context, see Birge, 1980).

Nevertheless, it would be interesting to have theoretical results similar to those derived in $\$ 4$ for familiar optimization of stochastic system problems in continuous time involving dynamics driven by semimartingales (see e.g. Shiryaev, 1980). The difficulty in attempting an analogue of the analysis presented in this paper for such systems is that the corresponding perturbed abstract problem (as utilized in \$4) must make sense. Put differently, the original stochastic optimization problem must remain well defined when nonanticipativity is relaxed. Using the Ito calculus approach (and its recent extensions to semimartingales generating mixed diffusion and jump dynamics) this 
is not possible, since the rigorous analytic integral form of the dynamics requires nonanticipativity of the integrand in the stochastic integrals involved. This technical requirement of the stochastic integration theories utilized has been relaxed for integration of Gaussian processes with respect to similar processes by Enchev and Stoyanov (1980), but this setting is of insufficient generality for many systems of interest. More promising is the application to the problem at hand of the recent pathwise theory of stochastic integration introduced for the study of stochastic differential equations whose integrals are driven by processes with continuous sample paths by Sussman (1978) and developed for semimartingales with jumps, for example, by Marcus (1981).

In the case of successful application of the approach of this paper to optimization of stochastic systems in continuous time, with differential dynamics in $\mathbb{R}_{\mathrm{n}}$ of the form $\underset{\sim}{\dot{x}}=\underset{\sim}{f}(\underset{\sim}{x})$, it may be conjectured that the full expected value of perfect information process $\underset{\sim}{\sigma}$ in $L_{1}$ may be recovered from the marginal EVPI process $\rho_{\sim}^{\prime}$ in $L_{1}^{n^{\prime}}$ by (Lebesque) integration as

$$
\underset{\sim t}{\sigma}=\int_{t}^{\tau} \underset{\sim}{\rho} \underset{\sim}{i} \underset{\sim s}{\dot{x}} \mathrm{ds}
$$

for an appropriate definition of $\underset{\sim}{\dot{x}}$. This is again a statement of some potential practical importance for stochastic system modelling.

\section{ACKNOWLEDGEMENTS}

I would like to express my gratitude to J-M. Bismut, who first pointed out to me the technical difficulties discussed in \$4 regarding extension of the present analysis to stochastic systems in continuous time, and to M.H.A. Davis, whose conversation lead to the suggestions made therein for surmounting them. 
REFERENCES

1. J. Birge (1981). Stochastic dynamic linear programs. Ph.D. Thesis Operations Research Department, Stanford University.

2. M. Brokate (1980). A regularity condition for optimization in Banach spaces: Counter examples. Appl.Math.Optim. 6, 189-192.

3. M.A.H. Dempster (1976). Lectures on Abstract Optimization and Its Applications. Department of Mathematics, Melbourne University.

4. M.A.H. Dempster (1980). Introduction to stochastic programming. In: Stochastic Programming. M.A.H. Dempster, ed. Acadernic, London. 3-59.

5. A.Y. Dubovitskii \& A.A. Milyutin (1965). Extremum problems in the presence of restrictions. USSR Comput.Math. \& Math.Phys. 5.3, $1-80$.

6. N. Dunford \& J.J. Schwartz (1956). Linear operators. Vol.I. Interscience, New York.

7. M.J. Eisner \& P. Olsen (1975). Duality for stochastic programming interpreted as L.P. in $\mathrm{L}_{\mathrm{p}}$-space. SIAM J.Appl.Math. 28, 779-792.

8. M.J. Eisner \& P. Olsen (1980). Duality in probabilistic programming. In: Stochastic Programming, op.cit., 147-158.

9. O.B. Enchev \& J.M. Stoyanov (1980). Stochastic integrals for Gaussian random functions. Stochastics 3, 277-289.

10. R.C. Grinold (1976). Manpower planning with uncertain demands. operations Res. $24,387-399$.

11. R.C. Grinold (1980). A class of constrained linear control problems with stochastic coefficients. In: Stochastic Programming, op.cit., 97-108.

12. J-B. Hiriart-Urruty (1978). Conditions necessaires d'optimalité pour un programme stochastique avec recours. SIAM J. Control optim. $16.2,317-329$.

13. J-B. Hiriart-Urruty (1981). Extension of Lipschitz integrands and minimization of nonconvex integral functionals: Applications to the optimal recourse problem in discrete time. Probability and Statistics 1, to appear.

14. F. Lempio \& H. Maurer (1980). Differential stability in infinitedimensional nonlinear programming. Appl.Math. Optim. 6, 139-152.

15. F. Louveaux \& Y. Smeers (1981a). A stochastic model for electricity generation. In: Modelling of Large-Scale Energy Systems. W. Haefele $\varepsilon$ L.K. Kirchmayer, eds. IIASA Proc.Series No.12. Pergamon, Oxford, 313-320.

16. F. Louveaux \& Y. Smeers (1981b). Optimization of a stochastic model for electricity generation. Stochastics. Special issue containing the Proceedings of the IIASA Task Force Meeting on Stochastic Optimization, 9-13 December, 1980. To appear. 
17. S.I. Marcus (1981). Modelling and approximation of stochastic differential equations driven by semimartingales. Stochastics 4.3, 223-246.

18. E.C. Prescott \& R.E. Lucas, Jr. (1972). A note on price systems in infinite dimensional space. Intl. Econ.Rev. 13, 416-422.

19. R.T. Rockafellar \& R.J-B. Wets (1976a). Stochastic convex programming: Relatively complete recourse and induced feasibility. SIAM J.Control optim. 14, 574-589.

20. R.T. Rockafellar \& R.J-B. Wets (1976b). Nonanticipativity and L ${ }^{1}-$ martingales in stochastic optimization problems. Math. Programming Studies 6, 170-187.

21. R.T. Rockafellar \& R.J-B. Wets (1978). The optimal recourse problem in discrete time: $L^{1}$-multipliers for inequality constraints. SIAM J.Control optim. 16.1, 16-36.

22. R.T. Rockafellar \& R.J-B. Wets (1981). Stochastic optimization problems of Bolza type in discrete time. Stochastics. IIASA Special Issue, op.cit. To appear.

23. A.N. Shiryayev (1980). Martingales: Recent Developments, Results, Applications. Lecture notes distributed at the 1980 European Meeting of Statisticians, Brighton, England.

24. H.J. Sussmann (1978). On the gap between deterministic and stochastic ordinary differential equations. Ann.Prob. 6.1, 19-41.

25. M. Valadier (1974). A natural supplement of $L_{1}$ in the dual of $L_{\infty}$. Seminaire d'Analyse Convexe, Montpelier.

26. A.F. Veinott (1966). The status of mathematical inventory theory. Management sci. 12, 745-777.

27. K. Yosida \& E. Hewitt (1952). Finitely additive measures. Trans. A.M.S. $72,46-66$.

28. J. Zowe \& S. Kurcyusz (1979). Regularity and stability for the mathematical programming problem in Banach spaces. Appl.Math. optim. 5, 49-62. 